\author{
Article \\ Doi 10.5943/mycosphere/8/7/7 \\ Copyright $(\mathcal{C}$ Guizhou Academy of Agricultural Sciences
}

\title{
A new species and a new combination of Aleurodiscus s.l. (Russulales, Basidiomycota)
}

\section{Dai LD and He SH*}

\author{
Institute of Microbiology, Beijing Forestry University, Beijing 100083, China
}

Dai LD, He SH. 2017 - A new species and a new combination of Aleurodiscus s.l. (Russulales, Basidiomycota). Mycosphere 8 (7), 908-916, Doi 10.5943/mycosphere/8/7/7

\begin{abstract}
Aleurodiscus ryvardenii sp. nov. from southwestern China is described and illustrated. The species is characterized by possessing effused basidiocarps, simple-septate generative hyphae, moniliform gloeocystidia and verrucose basidiospores. Aleurodiscus tsugae on coniferous bark in East Asia is transferred to Aleurocystidiellum. Illustrated description of Aleurocystidiellum tsugae and a key to the three species of Aleurocystidiellum are given. The phylogeny of 29 species of Aleurdiscus s.1., Stereum and Xylobolus was inferred from ITS sequences.
\end{abstract}

Key words - Aleurocystidiellum - ITS - Stereaceae - taxonomy - wood-inhabiting fungi

\section{Introduction}

Aleurodiscus s.l. is an important group of wood-decaying fungi including a large number of species with very different morphological characters (Núñez \& Ryvarden 1997). Although several new species and new records have been discovered in subtropical and tropical China (Dai \& He 2016, Dai et al. 2017a, b), the species diversity in these areas is still unclear. Recently, two specimens collected at Ailaoshan Nature Reserve, Yunnan Province, southwestern China is re-studied by morphological and molecular methods. They have totally effused basidiocarps, simple-septate generative hyphae, large moniliform gloeocystidia and strongly amyloid verrucose basidiospores, but are absent of acanthophyses. The effused yellow basidiocarps and large gloeocystidia resemble species of Megalocystidium Jülich which however differ in having clamped generative hyphae and smooth to minutely verrucose basidiospores. The species is described and illustrated as Aleurodiscus ryvardenii new to science.

Aleurocystidiellum P.A. Lemke was erected by Lemke (1964) to accommodate Aleurodiscus subcruentatus (Berk. \& M.A. Curtis) Burt which has discoid to stereoid basidiocarps, a dimitic hyphal system, encrusted skeletal cystidia and large verrucose basidiospores (Bernicchia \& Gorjón 2010). Tellería (1990) transferred Aleurodiscus disciformis (DC.) Pat. to Aleurocystidiellum. Morphologically, Aleurocystidiellum disciforme (DC.) Tellería has moniliform gloeocystidia rather than skeletocystidia in A. subcruentatum (Berk. \& M.A. Curtis) P.A. Lemke, but otherwise they are very similar. Meanwhile, the two-species formed a distinct lineage distant from Aleurodiscus s.s. in phylogenetic trees (Wu et al. 2001, Larsson \& Larsson 2003, Binder et al. 2005). Aleurodiscus tsugae was originally described from Japan on bark of Tsuga, and then found on Pinus in Japan, the Russian Far East and northeastern China (Maekawa 1993, Núñez \& Ryvarden 1997, Wu 2008). Careful 
morphological and molecular studies of the Chinese specimens of Aleurodiscus tsugae show that it belongs Aleurocystidiellum, and thus a new combination is proposed.

\section{Materials \& Methods}

Morphological studies - Voucher specimens are deposited at the herbarium of Beijing Forestry University (BJFC), Beijing, China. Samples for microscopic examination were mounted in Melzer's reagent or $1 \%$ phloxine, and the procedures followed Chen et al. (2016). The following abbreviations are used: $\mathrm{L}=$ mean spore length, $\mathrm{W}=$ mean spore width, $\mathrm{Q}=\mathrm{L} / \mathrm{W}$ ratio, $\mathrm{n}(\mathrm{a} / \mathrm{b})=$ number of spores (a) measured from number of specimens (b). Color names and codes are from Kornerup \& Wanscher (1978).

DNA extraction and sequencing - A CTAB plant genome rapid extraction kit-DN14 (Aidlab Biotechnologies Co., Ltd) was employed for DNA extraction and PCR amplification from dried specimens. The ITS region was amplified with the primer pair ITS5 and ITS4 (White et al. 1990) using the following procedure: initial denaturation at $95{ }^{\circ} \mathrm{C}$ for $4 \mathrm{~min}$, followed by 34 cycles at 94 ${ }^{\circ} \mathrm{C}$ for $40 \mathrm{~s}, 58{ }^{\circ} \mathrm{C}$ for $45 \mathrm{~s}$ and $72{ }^{\circ} \mathrm{C}$ for $1 \mathrm{~min}$, and final extension at $72{ }^{\circ} \mathrm{C}$ for $10 \mathrm{~min}$. DNA sequencing was performed at Beijing Genomics Institute, and the sequences were deposited in GenBank (Table 1).

Phylogenetic analyses - The molecular phylogeny was inferred from a dataset of 31 ITS sequences representing 24 Aleurodiscus s.l., 4 Stereum Hill ex Pers., one Xylobolus P. Karst and one outgroup species. Six sequences are newly generated, while others are mainly from Dai \& He (2016) and Dai et al. (2017a, Table 1). Heterobasidion parviporum Niemelä \& Korhonen was selected as outgroup (Dai \& He 2016). The sequences were aligned using ClustalX v.1.83 (Chenna et al. 2003). Alignments were optimized manually in BioEdit v.7.0.5.3 (Hall 1999), and then deposited at TreeBase (http://treebase.org/treebase-web/home.html, submission ID 21170).

Maximum parsimony analysis (MP) were performed using PAUP* 4.0b10 (Swofford 2002). Gaps in the alignments were treated as missing data. Trees were generated using 100 replicates of random stepwise addition of sequence and tree-bisection reconnection (TBR) branch-swapping algorithm, with all characters given equal weight. Branch supports for all parsimony analyses were estimated by performing 1000 bootstrap replicates (Felsenstein 1985) with a heuristic search of 10 random-addition replicates for each bootstrap replicate. The tree length (TL), consistency index (CI), retention index $(\mathrm{RI})$, rescaled consistency index (RC), and homoplasy index (HI) were calculated for each generated tree.

For Bayesian inference (BI), best models of evolution were obtained using MrModeltest v.2.2 (Nylander 2004), and posterior probabilities (BPP) were obtained by Markov Chain Monte Carlo sampling in MrBayes v.3.1.2 (Ronquist \& Huelsenbeck 2003). Four simultaneous Markov chains were run for five million generations for the dataset, and trees were sampled every 100th generation. The first quarter of the trees, which represented the burn-in phase of the analyses, were discarded, and the remaining trees were used to calculate posterior probabilities in the majority rule consensus tree.

\section{Phylogenetic Results}

The ITS dataset had an aligned length of 649 characters, of which 328 are parsimonyinformative. MP analysis yielded four equally parsimonious trees $(\mathrm{TL}=1456, \mathrm{CI}=0.530, \mathrm{RI}=0.630$, $\mathrm{RC}=0.334, \mathrm{HI}=0.470$ ). The best-fit evolution model for BI was "GTR $+\mathrm{I}+\mathrm{G}$ ". BI resulted in a topology similar to that of MP analysis with an average standard deviation of split frequencies = 0.006390 . The strict consensus MP tree is shown in Fig. 1 with both BT values $(\geq 50 \%)$ and BPPs $(\geq$ 0.95) labelled along the branches. In the tree, Aleurodiscus ryvardenii forms a distinct lineage. Aleurocystidiellum disciforme, A. subcruentatum and A. tsugae form a strongly supported clade (BT $=100 \%$, BPPs $=1.00$ ). 
Table 1 Species and sequences used in the phylogenetic analyses. Newly generated sequences are set in bold

\begin{tabular}{|c|c|c|c|}
\hline Taxa & Voucher & Locality & ITS \\
\hline Acanthobasidium bambusicola & He 2357 & China & KU559343 \\
\hline Acanthobasidium weirii & НHB 13132 & Italy & KX306882 \\
\hline Aleurocystidiellum disciforme & He 3159 & China & KU559340 \\
\hline Aleurocystidiellum tsugae & He 4167 & China & KY706209 \\
\hline Aleurocystidiellum tsugae & He 4025 & China & KY706211 \\
\hline Aleurocystidiellum subcruentatum & He 2886 & China & KU559341 \\
\hline Aleurodiscus amorphous & Ghobad-Nejhad 2464 & China & KU559342 \\
\hline Aleurodiscus aurantius & KC 565 & UK & HQ441573 \\
\hline Aleurodiscus botryosus & He 2712 & China & KX306877 \\
\hline Aleurodiscus canadensis & Wu 1207-90 & China & KY706203 \\
\hline Aleurodiscus cerussatus & He 3007 & China & KX306875 \\
\hline Aleurodiscus dextrinoideocerussatus & He 2820 & China & KY706206 \\
\hline Aleurodiscus effusus & He 2261 & China & KU559344 \\
\hline Aleurodiscus gigasporus & Wu 0108-15 & China & KY706205 \\
\hline Aleurodiscus grantii & He 2895 & China & KU559347 \\
\hline Aleurodiscus ljubarskii & He 2418 & China & KU559349 \\
\hline Aleurodiscus mesaverdensis & FP 120155 & USA & KU559359 \\
\hline Aleurodiscus mirabilis & He 3730 & China & KX306878 \\
\hline Aleurodiscus oakesii & He 2243 & USA & KU559352 \\
\hline Aleurodiscus penicillatus & НHB 14115 & USA & KU559338 \\
\hline Aleurodiscus ryvardenii & He 2648 & China & KX306879 \\
\hline Aleurodiscus tenuissimus & He 3575 & China & KX306880 \\
\hline Aleurodiscus tropicus & He 3833 & China & KX553875 \\
\hline Aleurodiscus wakefieldiae & He 2580 & China & KU559353 \\
\hline Heterobasidion parviporum & 091605 & China & KJ651503 \\
\hline Neoaleurodiscus fujii & He 2921 & China & KU559357 \\
\hline Stereum complicatum & He 2234 & USA & KU559368 \\
\hline Stereum ostrea & He 2067 & USA & KU559366 \\
\hline Stereum sanguinolentum & He 2111 & USA & KU559367 \\
\hline Stereum spectabile & $275-597$ & Japan & AB509853 \\
\hline Xylobous frustulatus & He 2231 & USA & KU881905 \\
\hline
\end{tabular}

\section{Taxonomy}

Aleurodiscus ryvardenii S.H. He \& Y.C. Dai, sp. nov.

MycoBank number: MB 821576; Facesoffungi number: FoF 03397

(Figs 2a, 3)

Type - China, Yunnan Province, Jingdong County, Ailaoshan Nature Reserve, alt. 2500 m, on dead angiosperm branch, 25 August 2015, He 2648 (BJFC 022006, holotype).

Etymology - Named in honor of Prof. Leif Ryvarden (University of Oslo, Norway), the author of the monograph of Aleurodiscus s.l.

Fruiting body - Basidiocarps annual, resupinate, effused, closely adnate, inseparable, membraneous to coriaceous, soft, up to $16 \mathrm{~cm}$ long, $2 \mathrm{~cm}$ wide, $0.2 \mathrm{~mm}$ thick. Hymenophore smooth, pale orange (6A3), greyish orange $[6 \mathrm{~B}(3-6)]$ to brownish orange $[6 \mathrm{C}(3-8)]$, not cracked; margin determinate, indistinct, concolorous or slightly darker than hymenophore surface. 


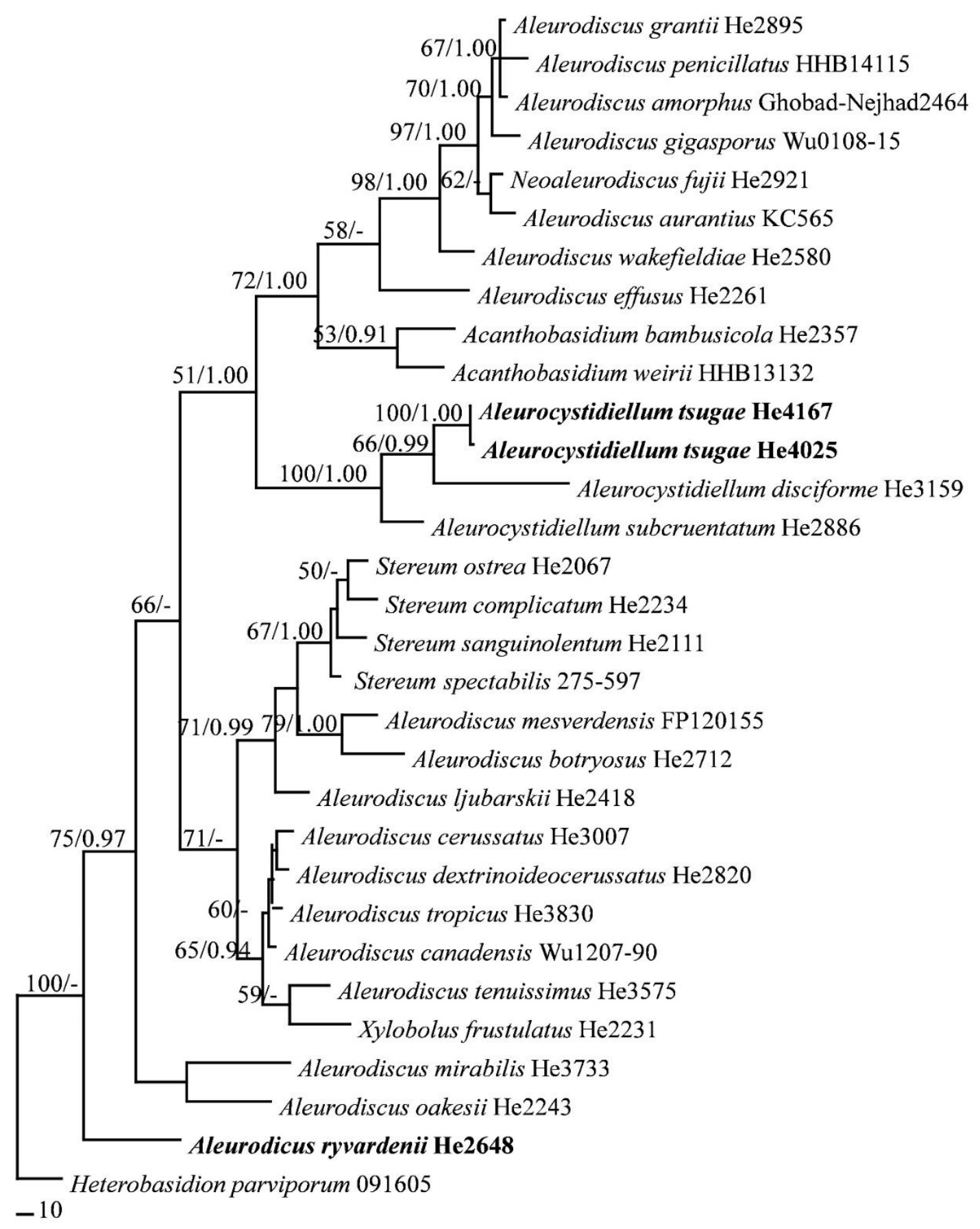

Figure 1 - Maximum parsimony strict consensus tree illustrating the phylogeny of species of Aleurodiscus s.l. and related genera based on ITS sequences. Branches are labelled with parsimony bootstrap values (before slash) $\geq 50 \%$ and Bayesian posterior probabilities (after slash) $\geq 0.95$.

Microscopic structure - Hyphal system monomitic. Subiculum indistinct to almost lacking. Generative hyphae abundant, simple-septate, thin- to slightly thick-walled, frequently septate and branched, usually constricted at the septa, mostly vertically arranged, more or less agglutinated, intermingled with gloeocystidia, 2.5-5.5 $\mu \mathrm{m}$ in diam. Gloeocystidia abundant, moniliform with several constrictions, tapering toward the apex, sinuous, easily broken, slightly thick-walled, 85-110 $(-160) \times 11-15(-18) \mu \mathrm{m}$. Simple hyphidia present, thin-walled, hyaline. Basidia subclavate to subcylindrical, hyaline, slightly thick-walled, with 4 sterigmata and a basal simple septum, 50-65 $\times$ 15-22 $\mu \mathrm{m}$; basidioles similar to basidia but smaller. Basidiospores broadly ellipsoid to subglobose, hyaline, thick-walled, verrucose, bearing an apiculus, strongly amyloid, $12-17(-18) \times 10-15(-16.5)$ $\mu \mathrm{m}, \mathrm{L}=14.5 \mu \mathrm{m}, \mathrm{W}=13.2 \mu \mathrm{m}, \mathrm{Q}=1.1(\mathrm{n}=60 / 2)$. 

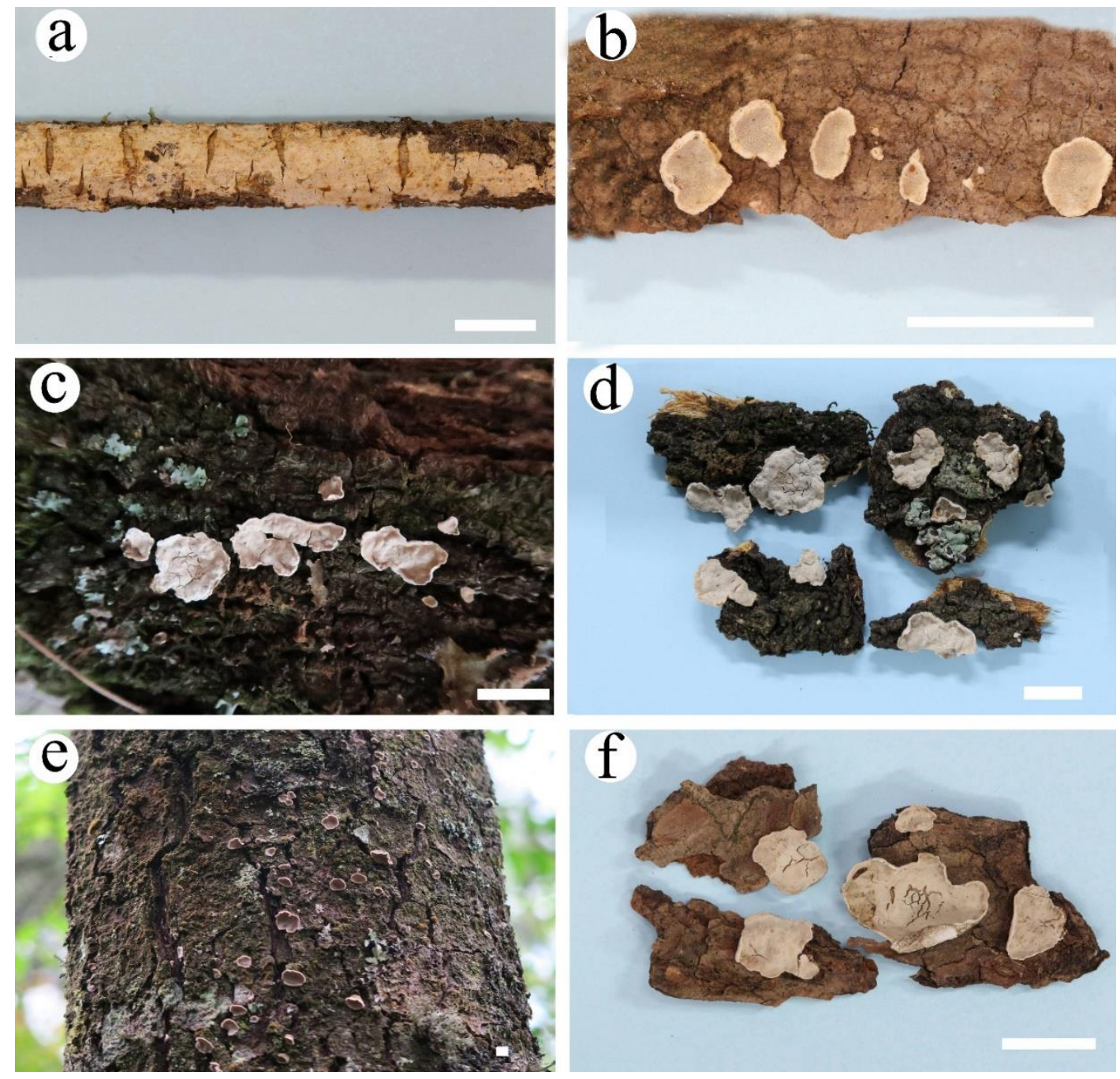

Figure 2 - Basidiocarps of Aleurodiscus s.l. species. a: Aleurodiscus ryvardenii (holotype, He 2648); b: Aleurocystidiellum subcruentatum (He 2886); c-d: Aleurocystidiellum disciforme (He 3159); e-f: Aleurocystidiellum tsugae (e: He 3734; f: He 2326). Scale bars $=1 \mathrm{~cm}$.

Additional specimen examined - China, Yunnan Province, Jingdong County, Ailaoshan Nature Reserve, alt. $2500 \mathrm{~m}$, on dead angiosperm branch, 25 August 2015, He 2652 (BJFC 021093, paratype).

Remarks: Although Aleurodiscus ryvardenii forms a distinct lineage distant from A. amorphus (Pers.) J. Schröt. (generic type) in the phylogenetic tree (Fig. 1), we place it in Aleurodiscus sensu Núñez \& Ryvarden (1997) according to morphological characters. Aleurodiscus ryvardenii is similar to A. cremicolor Hjortstam \& Ryvarden by sharing corticioid basidiocarps, simple-septate generative hyphae, numerous gloeocystidia and verrucose basidiospores. However, A. cremicolor has smaller gloeocystida $(40-60 \times 7-10 \mu \mathrm{m})$ and narrower basidiospores $(12-14 \times 6-8 \mu \mathrm{m}$, Núñez \& Ryvarden 1997). Aleurodiscus lacunae-equimortui J.A. Simpson \& Grgur. reported from Australia also has simple-septate generative hyphae and large gloeocystidia, but differs from A. ryvardenii by having numerous dendrohyphidia and larger basidiospores $(17-31.8 \times 15-21 \mu \mathrm{m}$, Simpson \& Grgurinovic 2003).

Aleurocystidiellum tsugae (Yasuda in Lloyd) S.H. He \& Y.C. Dai, comb. nov. MycoBank number: MB 821577; Facesoffungi number: FoF 03398

Basionym: Aleurodiscus tsugae Yasuda in Lloyd, Mycological Writings 6 (65): 1066, 1920.

Synonym: Acanthophysium tsugae (Yasuda) Parmasto, Eesti NSV Teaduste Akadeemia Toimetised 14 (2): 220, 1965. 


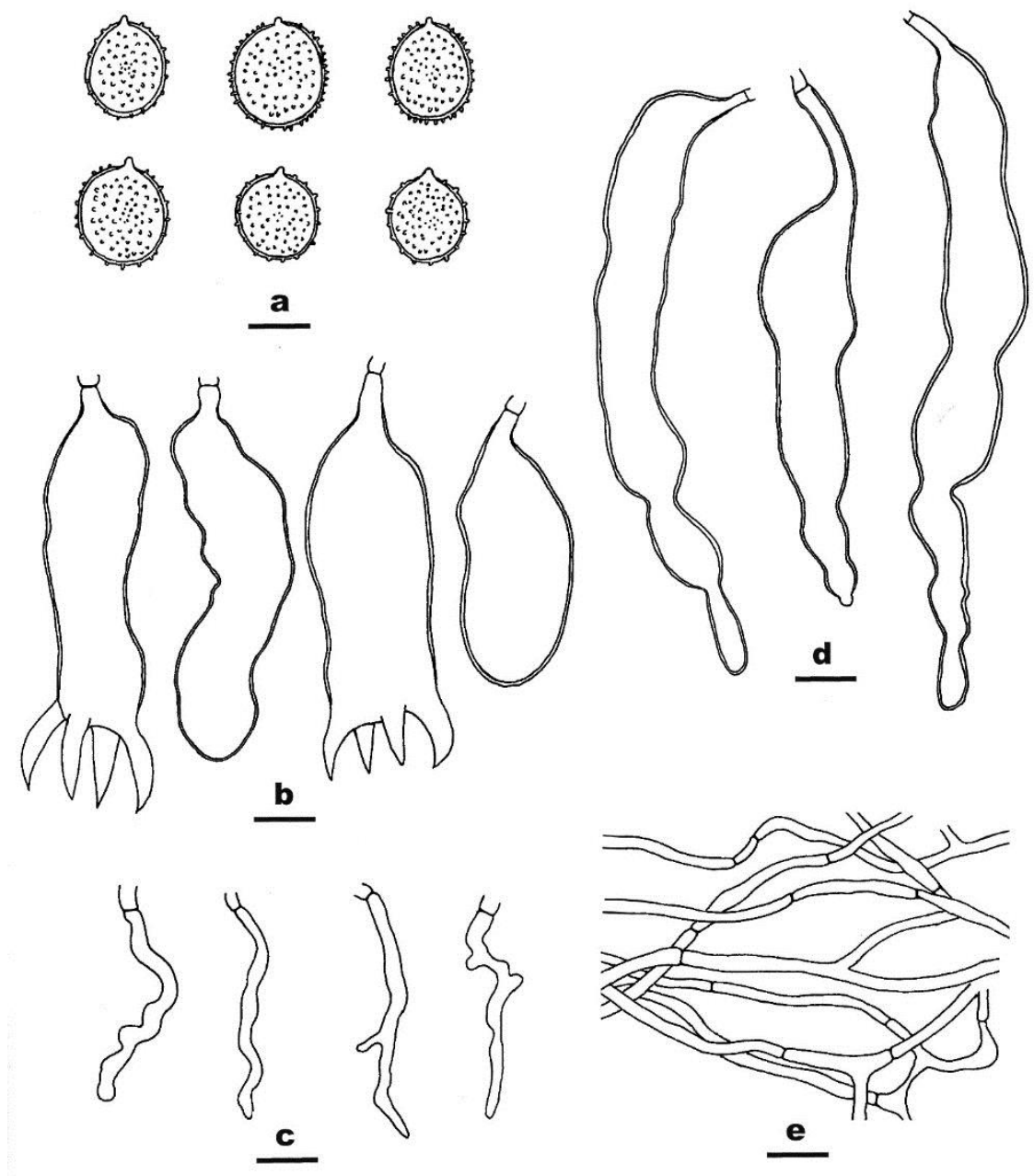

Figure 3 - Microscopic structures of Aleurodiscus ryvardenii (holotype, He 2648). a: Basidiospores; b Basidia and basidioles; c: Hyphidia; d: Gloeocystidia; e: Generative hyphae. Scale bars $=10 \mu \mathrm{m}$.

Fruiting body - Basidiocarps discoid, effused-reflexed to subpileate, easily detached from substrate, woody hard, effused parts up to $2.5 \mathrm{~cm}$ long, $1 \mathrm{~cm}$ wide, $0.1 \mathrm{~cm}$ thick, reflexed parts projecting up to $0.5 \mathrm{~cm}$. Abhymenial surface orange grey (6B2), grey [6(B-F)1)] to brownish grey (6F2); margin indistinct, sharp, curved inward, concolorous or slightly lighter than abhymenial surface. Hymenophore smooth, orange grey (6B2) to brownish grey [6(C-D)2], not cracked; margin thinning out, narrow, indistinct, slightly lighter than hymenophore surface.

Microscopic structure - Hyphal system monomitic. Subiculum thickening, generative hyphae in this layer thin- to thick-walled, hyaline, clamped, moderately branched and septate, sometimes distinctly thick-walled, very rarely branched and septate resembling skeletal hyphae, more or less regularly arranged, 2-6 $\mu \mathrm{m}$ in diam. Subhymenium thickening, composed of old hymenial elements, generative hyphae in this layer thin- to thick-walled, vertically arranged, frequently branched and septate, more or less agglutinated. Acanthophyses absent. Gloeocystidia in hymenium numerous, moniliform, slightly thick-walled, 80-150 × 10-13 $\mu \mathrm{m}$. Dendrohyphidia present, thin-walled, scattered. Basidia subclavate, slightly sinuous, hyaline, slightly thick-walled, with 4 sterigmata and a basal clamp connection, $60-100 \times 11-15 \mu \mathrm{m}$; basidioles similar to basidia but smaller. Basidiospores ellipsoid to broadly ellipsoid, hyaline, thick-walled, verrucose, bearing a distinct apiculus, strongly amyloid, 15-20 (-24) × 10-16 $\mathrm{m}, \mathrm{L}=17.5 \mu \mathrm{m}, \mathrm{W}=12.5 \mu \mathrm{m}, \mathrm{Q}=1.4(\mathrm{n}=$ 30/1). 

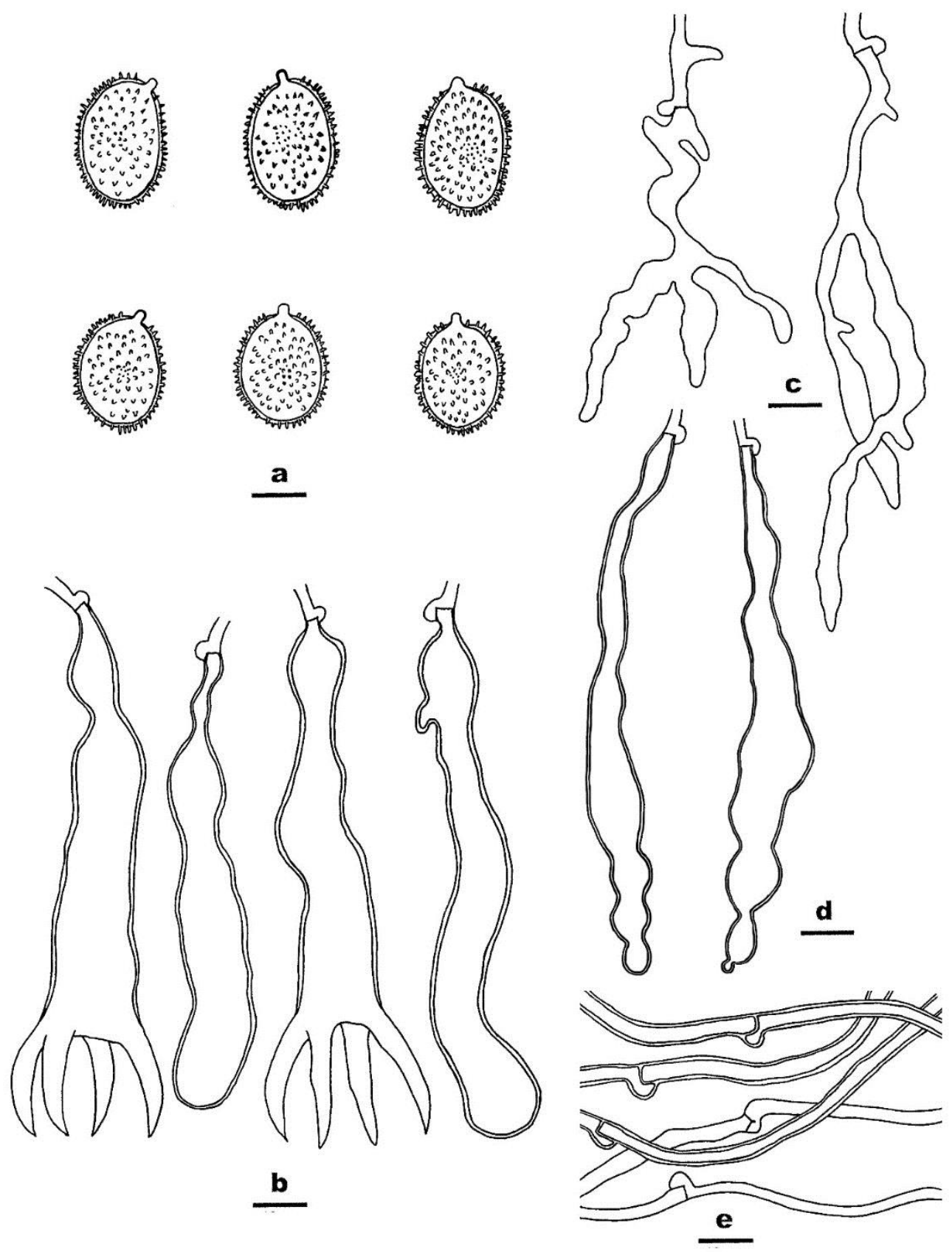

Figure 4 - Microscopic structures of Aleurocystidiellum tsugae (He 4024). a: Basidiospores; b: Basidia and basidioles; c: Dendrohyphidia; d: Gloeocystidia; e: Generative hyphae. Scale bars $=10$ $\mu \mathrm{m}$.

Specimens examined - Aleurocystidiellum tsugae: China, Guizhou Province, Leishan County, Leigongshan Nature Reserve, on bark of dead Pinus, 12 June 2016, He 3734 (BJFC 022233) \& He 3738 (BJFC 022237); Hunan Province, Liuyang County, Daweishan National Forest Park, on bark of dead Pinus, 10 July 2015, He 2326 (BJFC 020780); Jiangxi Province, Yifeng County, Guanshan Nature Reserve, 9 August 2016, He 4163 (BJFC 023605) \& He 4167 (BJFC 023609); Jilin Province, Fusong County, Lushuihe, on fallen trunk of Pinus, 19 August 2007, Wu 0708-16; Yunnan Province, Kunming, Xishan Park, on bark of dead Pinus, 1 August 2016, He 4022 (BJFC 023461); He 4023 (BJFC 023462 ); He 4024 (BJFC 023463); He 4025 (BJFC 023464). Aleurocystidiellum disciforme: China, Yunnan Province, Mouding County, Huafoshan Nature Reserve, on bark of living Quercus, 25 November 2015, He 3159 (BJFC 021554, Figs 2c-d). Aleurocystidiellum subcruentatum: China, Yunnan Province, Yulong County, Laojunshan Nature Reserve, alt. 3800 m, on bark of living Abies, 
31 August 2015, He 2877 (BJFC 021311), He 2879 (BJFC 021312), He 2886 (BJFC 022012, Fig. 2b), He 2894 (BJFC 021325), He2898 (BJFC 021329), He 2910 (BJFC 021341), He 2911 (BJFC 022013), He 2914 (BJFC 021344).

Remarks: Wu (2008) recorded Aleurocystidiellum tsugae in northeastern China, and we found that it also has a wide distribution in southern China on bark of Pinus. The species is similar to A. disciforme by sharing discoid to effused-reflexed basidiocarps, moniliform gloeocystida and verrucose basidiospores; however, the latter species has slightly smaller basidiospores $(15-17 \times 10$ $12 \mu \mathrm{m}$ ) and grows on angiosperms (Núñez \& Ryvarden 1997). In our phylogenetic analyses,

A. tsugae, A. disciforme and A. subcruentatum formed a fully supported clade (Fig. 1). Till now, all these three species of Aleurocystidiellum have been recorded in China (Dai 2011).

\section{Key to Aleurocystidiellum species:}

1. On angiosperms, mainly Quercus A. disciforme

1. On gymnosperms 2

2. Encrusted skeletocystidia present A. subcruentatum

2. Moniliform gloeocystidia present A. tsugae

\section{Acknowledgements}

Special thanks are due to Dr. Sheng-Hua Wu (National Museum of Natural Science, Taiwan) for specimen loans. We appreciate Prof. Yu-Cheng Dai (Beijing Forestry University, China) for his help in specimen collection and manuscript revision. The research was supported by the Fundamental Research Funds for the Central Universities (No. 2017PT09) and the National Natural Science Foundation of China (Nos. 31470144 \& 31670013).

\section{References}

Bernicchia A, Gorjón SP. 2010 - Fungi Europaei 12. Corticiaceae s.l. Edizioni Candusso, Alassio. pp. 1-1008.

Binder M, Hibbett DS, Larsson K-H, Larsson E, Langer E. 2005. The phylogenetic distribution of resupinate forms in the homobasidiomycetes. Systematics and Biodiversity 3, 113-157.

Chen JJ, Cui BK, Dai YC. 2016 - Global diversity and molecular systematics of Wrightoporia s.1. (Russulales, Basidiomycota). Persoonia 37, 21-36.

Chenna R, Sugawara H, Koike T, Lopez R et al. 2003 - Multiple sequence alignment with the Clustal series of programs. Nucleic Acids Research 31, 3497-3500.

Dai YC. 2011 - A revised checklist of corticioid and hydnoid fungi in China for 2010. Mycoscience $52,69-79$.

Dai LD, He SH. 2016 - New species and new records of Aleurodiscus s.l. (Basidiomycota) in China. Mycological Progress 15, 717-730.

Dai LD, Wu SH, Nakasone KK, Burdsall HH Jr., He SH. 2017a - Two new species of Aleurodiscus s.l. (Russulales, Basidiomycota) on bamboo from tropics. Mycoscience 58, 213-220.

Dai LD, Zhao Y, He SH. 2017b - Three new species of Aleurodiscus s.l. (Russulales, Basidiomycota) on bamboos from East Asia. Cryptogamie Mycologie (In press).

Felsenstein J. 1985 - Confidence intervals on phylogenetics: an approach using bootstrap. Evolution 39, 783-791.

Hall TA. 1999 - Bioedit: a user-friendly biological sequence alignment editor and analysis program for Windows 95/98/NT. Nucleic Acids Symposium Series 41, 95-98.

Kornerup A, Wanscher JH. 1978 - Methuen handbook of colour. 3rd edn. Eyre Methuen, London. pp $1-252$.

Larsson E, Larsson K-H. 2003 - Phylogenetic relationships of russuloid basidiomycetes with emphasis on aphyllophoralean taxa. Mycologia 95, 1037-1065.

Lemke PA. 1964 - The genus Aleurodiscus (sensu stricto) in North America. Canadian Journal of Botany 42, 213-282.

Maekawa N. 1993 - Taxonomic study of Japanese Corticiaceae (Aphyllophorales) I. Reports of the 
Tottori Mycological Institute 31, 1-149.

Núñez M, Ryvarden L. 1997 - The genus Aleurodiscus (Basidiomycotina). Synopsis Fungorum 12, $1-164$.

Nylander JAA. 2004 - MrModeltest v2. Program distributed by the author. Evolutionary Biology Centre, Uppsala University.

Ronquist F, Huelsenbeck JP. 2003 - MRBAYES 3: bayesian phylogenetic inference under mixed models. Bioinformatics 19, 1572-1574.

Simpson JA, Grgurinovic CA. 2003 - A new species of Aleurodiscus (Stereaceae) from Mt Kosciuszko, Australia. Australasian Mycologist 22, 15-19.

Swofford DL. 2002 - PAUP*: Phylogenetic analysis using parsimony (*and other methods). Version 4.0b10. Sinauer Associates, Sunderland, Massachusetts.

Tellería MT. 1990 - Annotated list of the Corticiaceae, sensu lato (Aphyllophorales, Basidiomycotina), for Peninsular Spain and Balearic Islands. Bibliotheca Mycologica 135, 1152.

White TJ, Bruns T, Lee S, Taylor J. 1990 - Amplification and direct sequencing of fungal ribosomal RNA genes for phylogenetics. In: Innis MA, Gelfand DH, Sninsky JJ, White TJ (eds.), PCR Protocols: A guide to methods and applications. Academic Press, San Diego, pp 315-322.

Wu SH. 2008 - Twenty species of corticioid fungi newly recorded from China. Mycotaxon 104, 7988.

Wu SH, Hibbett DS, Binder M. 2001 - Phylogenetic analyses of Aleurodiscus s.l. and allied genera. Mycologia 93, 720-731. 